\title{
Special Issue on the 57th International Midwest Symposium on Circuits and Systems
}

\author{
Jose Silva-Martinez $^{1}$ - Aydin İlker Karşılayan ${ }^{1} \cdot$ Jiang Hu $^{1} \cdot$ Harish Krishnaswamy $^{2}$
}

Published online: 21 June 2016

(C) Springer Science+Business Media New York 2016

This special issue contains extended papers of the 57th International Midwest Symposium on Circuits and Systems, selected by the Guest Editors based on reviewers' comments, attendees paper grading and session chair recommendations. The International Midwest Symposium on Circuits and Systems is the oldest Circuits and Systems Symposium sponsored by the IEEE CAS Society. This conference contributes to its strong history by reporting the latest research results and innovations in the field of circuits and systems through Distinguished Speakers featuring the newest innovations relevant to this field and shedding light on its evolution towards breaching the gaps among technologies. MWSCAS-2014 received 336 paper submissions from 32 countries. The papers were evaluated by a minimum of three reviewers. Among all these contributions, a subset of these papers were invited for this special issue. The invited papers went through a peer review process consisting of world recognized experts in related fields. After a long journey, 15 papers were finally accepted.

In the first paper, Tripurari et al., propose a wide-band RF channelizer architecture that splits the 0.6-9 GHz input spectrum in seven channels of $1.2 \mathrm{GHz}$ each. The

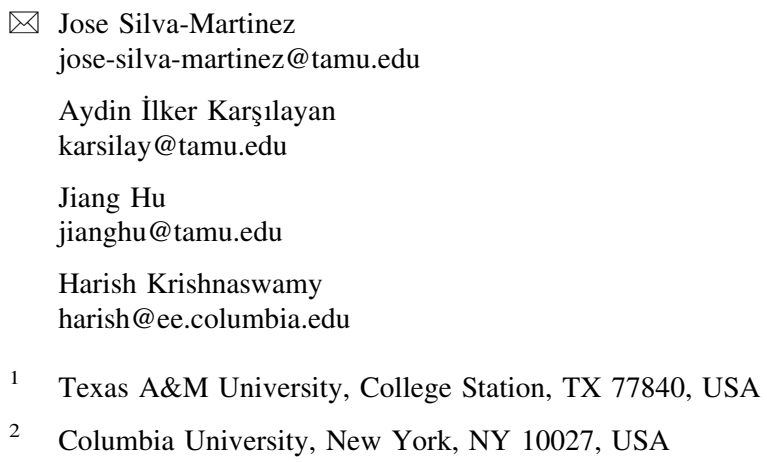

architecture enables multi-Gbps aggregate data reception with the property of agile switching between channels. The average power consumption is only $435 \mathrm{~mW}$ while achieving a dynamic range between 58 and $63 \mathrm{~dB}$. Two compact switchless dual-band load networks for class-E power amplifier (PA) operating at 800 and $1900 \mathrm{MHz}$ are reported by $\mathrm{Li}$ et al. The PA with transformer-based load network achieves an outstanding power added efficiency of $68.6 \%$ at low band and $62.6 \%$ at high band at an output power of 37.8 and $36.7 \mathrm{dBm}$, respectively. The LC-based PA shows a similar PAE of 68.3 and $60 \%$ at low band and high band, respectively. A MIMO satellite communication system with accelerated dual paths (ADP) asynchronous design authored by Che et al., reports an asynchronous design approach for multiple input multiple output satellite communication systems. Authors employ an ADP design; the data flow between the two paths, the increase the reliability of the system by circumventing transient faults induced delay. The proposed design can decrease the delay overhead of the entire system from 43.5 to $19.8 \%$ at the fault rate of 400/clock cycle. Ming et al., present a receiveside circuitry that merges the cancellation of both near-end and far-end crosstalk (NEXT/FEXT) and can automatically adapt to different channel environments. The techniques are demonstrated in a prototype fabricated in a $65-\mathrm{nm}$ CMOS process demonstrating effective crosstalk cancellation at $10 \mathrm{~Gb} / \mathrm{s}$.

Optimal two-stage comb decimators are reported by Salgado et.al. Authors show how to choose the decimation factor of the first stage in order to get simultaneously the best possible power reduction with optimal area tradeoff, in a comparison with a single cascade integrator-comb architecture. The issues related to susceptibility to supply noise in class D amplifiers are analyzed by $\mathrm{He}$ and coauthors. In this paper, the class D PSRR is analytically 
investigated; the analysis is applied to the ubiquitous 3-state Bridge-tied-load (BTL) closed-loop PWM CDA. The derived analytical expressions are verified by means of HSPICE simulations and on measurements on discretelyrealized CDAs. Damera et al., propose the use of minimally invasive filters. third order and fourth order filters are designed in $130 \mathrm{~nm}$ technology; when compared with the conventional filter implementations such as a Tow-Thomas architecture, the proposed third order solution achieves a total in-band input-referred integrated noise of $44 \mu \mathrm{V}$ compared to $79 \mu \mathrm{V}$ achieved by a Tow-Thomas implementation. In addition, lower power consumption is claimed. Soto Aguilar and coauthors introduce a methodology to reduce the distortion introduced by the harmonics of the clock in the operation of switched-R-MOSFET-C filters. The clock harmonic cancellation technique shows an improvement of more than $38 \mathrm{~dB}$ without compromising the tuning range or requiring increasing the frequency of the clock. The techniques are demonstrated employing a standard CMOS IBM $180 \mathrm{~nm}$. Chang and Onabajo report an instrumentation amplifier architecture with a mechanism that generates negative capacitances at its input that allows adaptive cancellation of the input capacitances from the electrode cables and printed circuit board. The proposed negative capacitance generation technique can improve the input impedance. An operational transconductance amplifier that achieves a transconductance of $25 \mathrm{pS}$ and very high output impedance is described.

Power management is another relevant area that is becoming more and more relevant. Cong and Lee report a techniques that promises to improve the power efficiency for high-voltage DC-DC converters employing high switching frequencies. A two-phase quasi-square-wave zero-voltage-switching (PS-TPZVS) cell is used to realize ZVS operation for all power FETs. The proposed design saves one auxiliary inductor and one auxiliary capacitor and thus reduces both the volume and power losses of the auxiliary circuitry. It is verified through simulations that peak efficiencies over $95 \%$ are feasible. Teh et al., report an energy harvesting scheme that generates bipolar output voltage $( \pm 1 \mathrm{~V})$. The architecture is based on a miniature 1:1 turn-ratio pulse transformer boost converter using subthreshold level input voltage source. Designed in a standard CMOS $0.13-1 \mathrm{~m}$ technology, chip chi functionality is verified. At start-up, the system only requires minimum start-up input voltage of $36 \mathrm{mV}$ at input power of $5.8 \mu \mathrm{W}$. A $0.75 \mathrm{~V}$ supply nanowatt resistorless sub-bandgap CMOS voltage reference is reported by Caicedo et al. The circuit uses a curvature compensation technique that allows higher temperature stability. Simulation results in $180 \mathrm{~nm}$ CMOS process, for a voltage of $469 \mathrm{mV}$ shows a temperature coefficient of $5 \mathrm{ppm} / \mathrm{C}$ for the $-40-125{ }^{\circ} \mathrm{C}$ extended temperature range. A microwatt voltage doubler-based voltage regulator for ultra-low power energy harvesting systems is reported by J. Salomaa et al. The architecture is based on switched-capacitor techniques and produces a stable $1.2-\mathrm{V}$ power supply using inputs from 0.63 to $1.8 \mathrm{~V}$. Implemented in a $180 \mathrm{~nm}$ CMOS process, the regulator achieves a regulator peak power and current efficiency are 63 and $49 \%$, respectively. Ture et al., developed a low-power pulse position modulation demodulator intended for remotely powered battery-less implant-table devices. The power for the implantable device is provided by the magnetic coupling employing external coils. Fabricated in a $0.18 \mathrm{~lm}$ CMOS technology, the experimental results verify the functionality of the PPM demodulator.

In the last paper, Shi et al., propose an optimization based on genetic algorithm in order to select the most suitable speed-sensors for speed binning. Authors demonstrate that optimizing sensor selection can improve speedbinning accuracy. The optimization algorithm shows improving accuracy beyond 94 and $93 \%$, respectively.

The Editors would like to recognize the support of the Organizing Committee of the 57th MWSCAS, Conference Steering Committee, Session Chairs, anonymous reviewers and special thanks to the authors. We hope you will enjoy reading these papers and find them useful for your future research endowments.

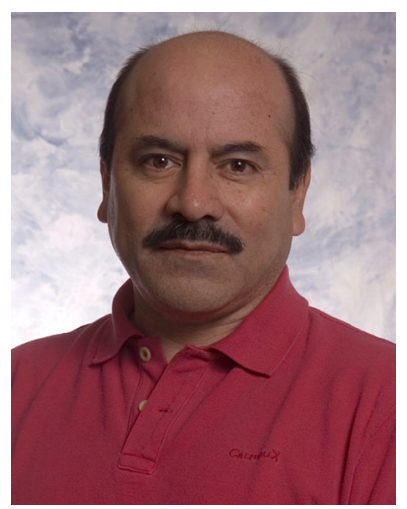

Jose Silva-Martinez (SM'98F'10) was born in Tecamachalco, Puebla, México. He received the M.Sc. degree from the Instituto Nacional de Astrofísica Optica y Electrónica (INAOE), Puebla, México, in 1981, and the Ph.D. degree from the Katholieke Univesiteit Leuven, Leuven Belgium in 1992. In 1993, he joined the Electronics Department, INAOE, and from May 1995 to December 1998, was the Head of the Electronics Department; He was a co-founder of the Ph.D. program on Electronics in 1993. He is currently with the Department of Electrical and Computer Engineering of the Texas A\&M University, at College Station, where he holds the position of Texas Instruments Professor. He is currently serving as the Associate Department Head for Graduate Student Affairs of the Department of Electrical and Computer Engineering at TAMU. He has published over 112 and 170 Journal and conference papers, respectively, 2 books and 12 book chapters, 1 granted patent and 5 more filed. His current field of research is in the areas of design and fabrication of integrated circuits for communication, radar and biomedical applications. Dr. Silva-Martinez served as the 2014-2015 Editor in Chief of the IEEE Transactions on Circuits and System PartII and was the Conference Chair of MWCAS-2014, he was a member of the DLP program of CASS 2013-2014 and was a senior editorial board member of IEEE JETCAS 2014-2015. He has served as IEEE CASS Vice President Region-9 (1997-1998), and as Associate Editor for IEEE Transactions on Circuits and Systems part-II during the 
periods 1997-1998 and 2002-2003, Associate Editor of IEEE TCAS Part-I 2004-2005 and 2007-2008, and currently serves in the board of editors of other three major journals. He is the recipient of the 2005 Outstanding Professor Award by the ECE Department, Texas A\&M University, co-author of the papers that received the MWCAS 2011 and RF-IC 2003 Best Student paper awards and recipient of the 1990 European Solid-State Circuits Conference Best Paper Award.

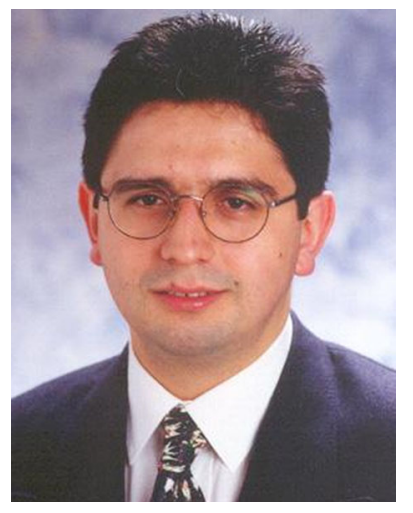

Aydın İker Karşılayan received the B.S. and M.S. degrees in Electrical Engineering from Bilkent University, Ankara, Turkey, in 1993 and 1995, respectively, and the Ph.D. degree from Portland State University, Portland, OR, USA, in 2000. He joined the faculty of Texas A\&M University, College Station, TX, USA, in 2000, where he is currently an Associate Professor of Electrical and Computer Engineering. His current research interests include high-frequency analog filters, data converters, automatic tuning, mixed-mode integrated circuit design, RF communication circuits, and power harvesting. Dr. Karşılayan served as an Associate Editor of the IEEE Transactions on Circuits and Systems I: Regular Papers, from 2002 to 2004, and the IEEE Transactions on Circuits and Systems II: Express Briefs from 2006 to 2010. He also served as an organizing committee member of the IEEE MWSCAS 2014.

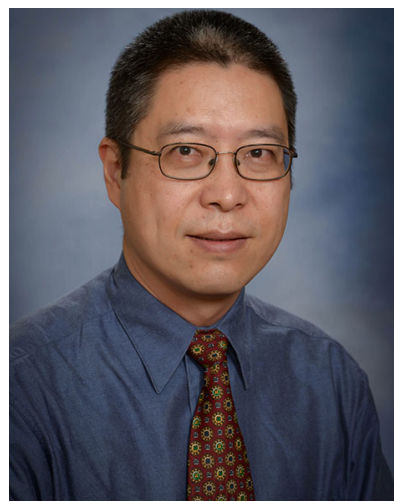

Jiang Hu received the B. S. degree in Optical Engineering from Zhejiang University, China, in 1990, the M. S. degree in physics in 1997, and the $\mathrm{Ph}$. D. degree in Electrical Engineering from the University of Minnesota in 2001. He was with IBM Microelectronics from January 2001 to June 2002. Currently he is a professor in the Department of Electrical and Computer Engineering at Texas A\&M University. His research interests include VLSI circuit optimization, chip power management, approximate computing and hardware security. He received a best paper award at the ACM/IEEE Design Automation Conference in 2001, an IBM Invention Achievement Award in 2003, and a best paper award at the IEEE/ ACM International Conference on Computer-Aided Design in 2011. He has served as General Chair and Technical Program Chair for the ACM International Symposium on Physical Design, and associate editor for IEEE Transactions on CAD and ACM Transactions on Design Automation of Electronic Systems. He was named an IEEE Fellow in 2016.

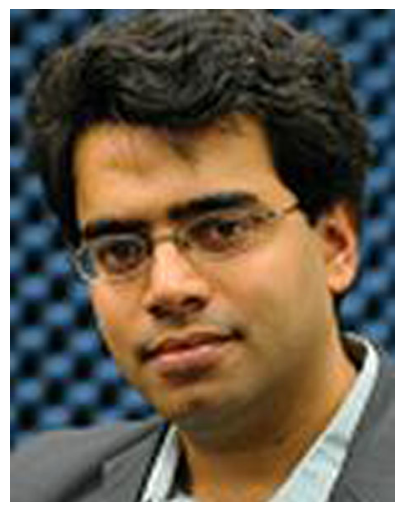

Harish Krishnaswamy received the B.Tech. degree in Electrical Engineering from the Indian Institute of Technology, Madras, India, in 2001, and the M.S. and Ph.D. degrees in Electrical Engineering from the University of Southern California (USC), Los Angeles, CA, USA, in 2003 and 2009, respectively. In 2009, he joined the Electrical Engineering Department, Columbia University, New York, NY, USA, where he is currently an Associate Professor. His research interests broadly span integrated devices, circuits, and systems for a variety of RF, mmWave and sub-mmWave applications. Dr. Krishnaswamy serves as a member of the Technical Program Committee (TPC) of several conferences, including the IEEE International Solid-State Circuits Conference (2015/16-present) and IEEE RFIC Symposium (2013-present). He was the recipient of the IEEE International Solid-State Circuits Conference (ISSCC) Lewis Winner Award for Outstanding Paper in 2007, the Best Thesis in Experimental Research Award from the USC Viterbi School of Engineering in 2009, the Defense Advanced Research Projects Agency (DARPA) Young Faculty Award in 2011, a 2014 IBM Faculty Award and the 2015 IEEE RFIC Symposium Best Student Paper Award-1st Place. 